\title{
Wavelet Transformation Application on Accommodation Equipments' Fault Diagnosis
}

\author{
Baoji YUAN* \\ College of Navy Submarine, \\ Qingdao, Shandong, P.R.China \\ E-mail: 18661780382@163.com \\ +* Corresponding author
}

\author{
Yanfeng WU \\ College of Navy Submarine, \\ Qingdao, Shandong, P.R.China \\ E-mail: 15066792976@163.com
}

\author{
Weining LI \\ College of Navy Submarine, \\ Qingdao, Shandong, P.R.China \\ E-mail: 1918808552@qq.com
}

\begin{abstract}
The submarine aircraft has several accommodation electrical equipments that have varieties of failure modes. The centralized control system includes accommodation electric network equipments' fault diagnosis and exception handling, so it is very important. With accommodation electric network scale expanding, the traditional fault processing way based on artificial experiences is kittle. Advanced fault identification and processing methods have been proposed to realize on-line fault processing with more fault data. The wavelet analysis is conductive to raise the fault processing working and shorten the deal time.
\end{abstract}

Keywords-fault diagnosis; wavelet analysis; equipment abnormal observation; collective control system

\section{INTRODUCTION}

Presently, underwater aircrafts have many accommodation electrical equipments [1] which are operated by complicated process including power cut-on or cut-off, and repair application. When the equipment fault emerges, a large amount of data needs to be handled by electricians [10]. The accommodation electricians need possess higher technical ability and rich experience [2]. Or else, they are unable to deal with several warning message and fault handling in short time. This is an urgently problem at present.

\section{AuXILIARy Decision-MAKING Key TeChNology ABOUT EQUIPMENT FAULT HANDLING}

Electric equipment fault handling function [3, 12] consists of fault on-line diagnose and abnormal state prewarning. Equipment fault handling function runs based on several switch states of monitoring system, abnormal states of electric parameters and prewarning information. With expert consulting system [9, 13, 15], it may be realized that faults of electric power networks are handled on time.

\section{EQUIPMENT FAULT DiAgNOSE ON-LINE}

Electric equipment fault handling system is expressed by Fig 1 as follow. Firstly, the system applies heuristic search method to get the net of doubtful components based on electric monitoring data. Then through checking all of doubtful components and judging by every premonitoring signal, the questionable component can be defined based on expert knowledge base [14]. Or else, the next doubtful component should be checked by the system until all of doubtful components are checked up seriously. Further, it is next to find the cause of defined fault component with monitoring information. For example, circuit switch deciliter appears shutting(compression force low, operating mechanism's fluid pressure low), voltage changer or electric reactor's protection acting, switch malfunction's protection acting, capacitor disbalance 's protection acting, complement shutting's protection acting and so on. The fault component should be isolated immediately according to the above prewarning signals. At the same time, if the condition of reset energizing is satisfied, the auxiliary decision-making and isolated handling plan should be given with equipment fault management process. 


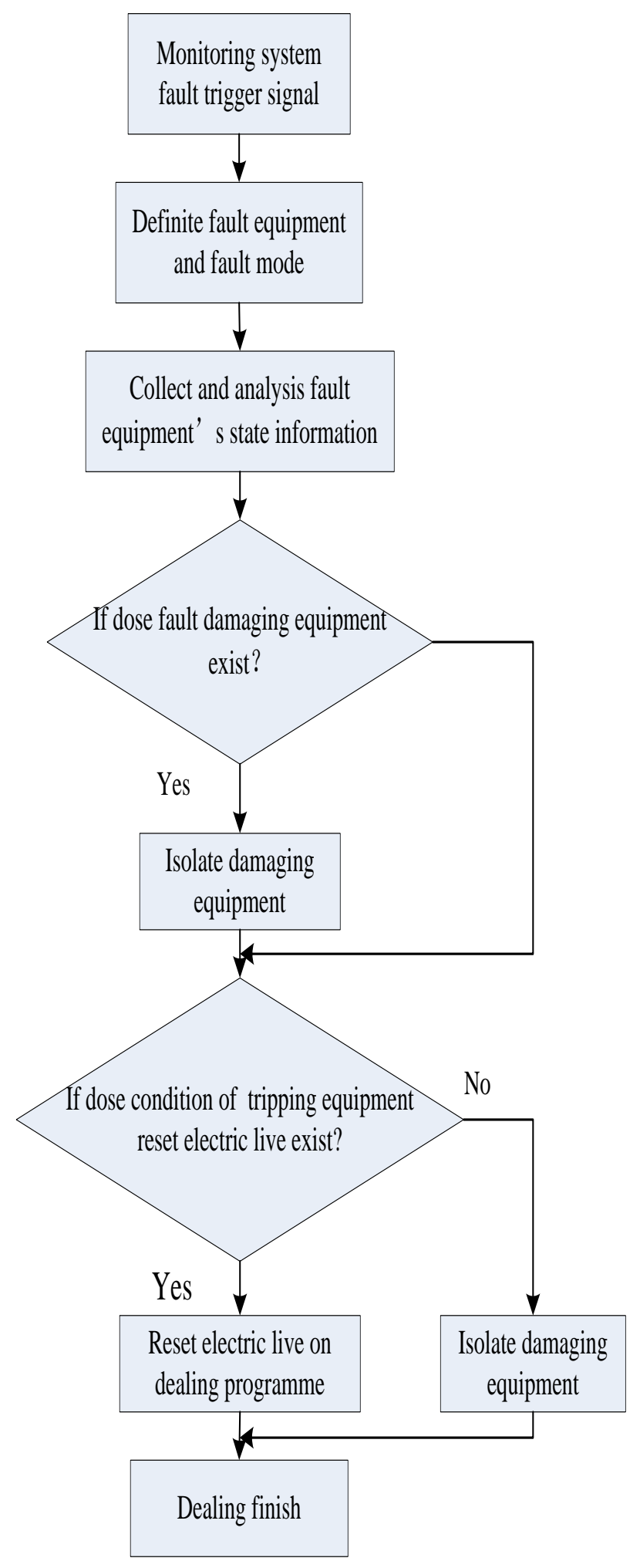

Figure 1. Equipment fault auxiliary decision-making analysis schema

\section{WaVElet ANALysis ON-Line MONITORING METHOD ON EQUIPMENT ABNORMAL STATE}

Wavelet analysis method [4] is the way to partially analysis the signal in time scale, and its time window and frequency window can be changed all in time domain. The low frequency part has higher frequency-resolution and lower time-resolution; on the contrary, the high frequency part has lower frequency-resolution and higher time-resolution. So this method extremely satisfies distinguishing the abnormal signal in normal message and bring up the fault component [11, 16], and it can be addressed as microscope of signal analysis. In accommodation equipments' fault diagnose, wavelet analysis method has favourable effect on electric parameters and state information.

In electric equipment's fault signal analysis, the frequency feature of signal in every time is vital, so advanced method should combine time-domain and frequency-domain to describe the feature of fault signal [5]. Gladly, wavelet analysis method can deal with these problems. It has fixed window area, but the size and shape of time window and frequency window can be changed [6].

Supposing $\psi(t) \in L^{2}(R), \psi(t)$ is converted to $\psi(w)$ by Fourier transform, $L^{2}(R)$ is square-integrable space. When $\psi(w)$ satisfies the following condition,

$$
C_{\psi}=\int_{R} \frac{\psi(w)}{|w|} d w<\infty
$$

$\psi(t)$ is a elemental wavelet function. Dilating and translating $\psi(w)$, we can get a wavelet array.

Under the continuous condition, wavelet array becomes

$$
\psi_{a, b}(t)=\frac{1}{\sqrt{a}} \psi\left(\frac{t-b}{a}\right), a, b \in R, a \neq 0
$$

Where $a$ is dilating agent, and $b$ is translating agent.

The continuous wavelet transform of any function $f(t) \in L^{2}(R)$ is

$$
W_{f}(a, b)=\left\langle f, \psi_{a, b}\right\rangle=a^{-1 / 2} \int_{R} f(t) \psi\left(\frac{t-b}{a}\right) d t
$$

$W_{f}(a, b)$ is described as wavelet transform of $f(t)$. 


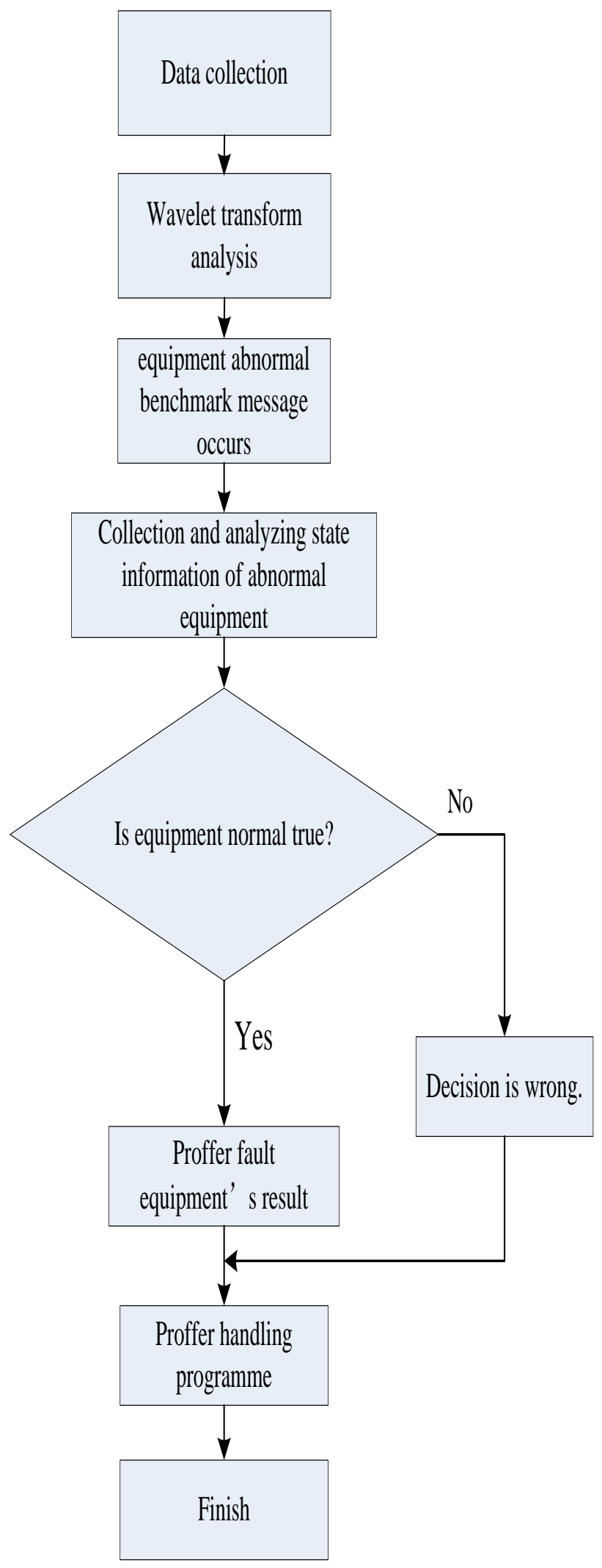

Figure 2. Equipment abnormal state analysis procedure by wavelet transform method

\section{Wavelet Transform Performance of White NOISE}

Suppose $n(x)$ is a flat white noise with real variance $\sigma^{2}$, the self-correlation function of $n(x)$ is

$$
R_{n}(u, v)=E[n(u) n(v)]=\delta(u-v) \sigma^{2}
$$

Suppose wavelet transform of $n(x)$ is $W_{n}(a, x)$, it is random process of $X$, and existing

$$
\left|W_{n}(a, x)\right|^{2}=\int_{-\infty}^{\infty} \int_{-\infty}^{\infty} n(u) n(v) \psi(x-u) \psi(x-v) d u d v
$$

Calculate the mathematical expectation of above equation, and we get

$$
\begin{aligned}
E\left(\left|W_{n}(a, x)\right|^{2}\right) & =\int_{-\infty}^{\infty} \int_{-\infty}^{\infty} \sigma^{2} \delta(u-v) \psi(x-u) \psi(x-v) d u d v \\
& =\frac{\|\psi\|^{2} \sigma^{2}}{a}
\end{aligned}
$$

Above equation express that wavelet transform's module square is inversely proportional to scale $a$ of white noise. Immediately, the scale is larger indicating component of noise lower which is important criterion of judging electric equipments' fault existence and fault mode [7]. At all, firstly we find abnormal phenomenon of electric equipment by computing Lipschitz exponent [8] $\alpha$ and smoothing factor $\sigma$, and then confirm fault cause by traditional methods based on the experience of electrical engineers.

\section{CONCLUSIONS}

Because underwater aircraft has several electric equipments, and several fault models, so it is very difficult to abstract fault feature. Lots of transient signals in electric equipment monitoring data, and frequency feature of any time is very important But dealing with these signals only in time-domain or only in frequency-domain is not enough. Considering that wavelet transform has local feature simultaneously in time-domain and frequency-domain, this paper has applied wavelet analysis transform technology to definite fault cause of electric equipments in accommodation. Firstly we find abnormal phenomenon of electric equipment by computing Lipschitz exponent $\alpha$ and smoothing factor $\sigma$ which can express emergent feature, and we make use of wavelet denoise technology to deal with noises of on-line monitoring data. Further, we confirm fault cause by traditional methods. A t last, a promoted programme has been proposed for electric equipment monitoring on-line.

\section{REFERENCES}

[1] V.H. Ferreira, R. Zanghi, M.Z. Fortes , et al. A survey on intelligent system application to fault diagnosis in electric power system 
transmission lines[J]. Electric Power Systems Research,2016,136(7): 135-153.

[2] [2] Ren Hui, Zhao Hong-Shan, Mi Zeng-Qiang,et al. Power system fault diagnosis by use of encoded Petri net models[J]. Power System Technology,2004,28(5):64-68.

[3] [3] Sun Mingwei, Tong Xiaoyang, Liu Xinyu,et al. A power system fault diagnosis method using temporal Bayesian knowledge bases[J]. Dianwang Jishu/Power System Technology,2014,38(3):715-722.

[4] [4] Momoh James A, Oliver Jr, Walter E,et al. Comparison of feature extractors on DC power system faults for improving ANN fault diagnosis accuracy[J]. Proceedings of the IEEE International Conference on Systems,1995,4:3615-3623.

[5] [5] Seo Gyu-Seok, Baek Young-Sik, Kim Jung-Nyun,et al. On-line fault diagnosis in the large power system[J]. IFAC Proceedings Volumes,2005,16: 285-290.

[6] [6] Jain M. Babita, Jain Amit, Srinivas M.B,et al. A web based expert system shell for fault diagnosis and control of power system equipment[J]. Proceedings of 2008 International Conference on Condition Monitoring and Diagnosis, CMD 2008, 2007: 1310-1313.

[7] [7] Zheng Haibo, Li Zhiyuan, Chen Xinzhao. Fault diagnosis for gearbox gear based on continuous wavelet transform[J]. Jixie Gongcheng Xuebao/Chinese Journal of Mechanical Engineering,2002,38(3):69-73.

[8] [8] Ye Hao, Wang Guizeng, Fang, Chongzhi. Wavelet transform based fault diagnosis for missile transporting vehicle[J]. Zidonghua Xuebao/Acta Automatica Sinica,1998,24:301-305.

[9] [9] Wu Jian-Da, Kuo Jun-Ming. An automotive generator fault diagnosis system using discrete wavelet transform and artificial neural network[J]. Expert Systems with Applications,2009,36(6): 9776-9783.

[10] [10] Zhang Xu, Zhao Dongmei, Li, Sheng. Research of power system fault diagnosis method[J]. 2009 International Conference on Energy and Environment Technology, ICEET 2009,2009,2:213-216.

[11] [11] WANG Lei,CHEN Qing,GAO Hongyu, et al.Model for And Tree in Power System Fault Diagnosis[J]. Automation of Electric Power Systems,2016,40(2):100-106.

[12] [12] Ferreira V.H, Zanghi R, Fortes M.Z,et al. A survey on intelligent system application to fault diagnosis in electric power system transmission lines[J]. Electric Power Systems Research,2016,136(7):135-153.

[13] [13] Yang Xiu-Wen, Chen Jie, Zeng Shun-Peng,et al. Application research of continuous wavelet transform in crack fault diagnosis of transmission gear[J]. Proceedings of the International Computer Conference 2006 on Wavelet Active Media Technology and Information Processing, ICWAMTIP 2006,2006: 1161-1166.

[14] [14] Mao Yongfang, Qin Yi, Tang Baoping,et al. Fault diagnosis of bearing based on overcomplete rational wavelet transform[J]. Journal of Vibration, Measurement and Diagnosis,2011,31(5): 626-630.

[15] [15] Li Hui. Bearing fault diagnosis based on time scale spectrum of continuous wavelet transform[J]. Proceedings - 2011 8th International Conference on Fuzzy Systems and Knowledge Discovery, FSKD 2011,2011,3: 1934-1937.

[16] $\mathrm{Li}$ Hui. Impulse response wavelet transform based bearing fault diagnosis[J]. Advanced Research on Industry, Information System and Material Engineering,2011, 204:1018-1021 